\title{
PARALLELIZABILITY AND FLAT MANIFOLDS ${ }^{1}$
}

JOHN A. THORPE

It is well known that every simply connected flat Riemannian manifold is parallelizable. The object of this paper is to improve this result by weakening the assumption of simple connectivity.

Given an orientable flat Riemannian manifold $X$ we find a condition on the holonomy group of $X$ sufficient to guarantee parallelizability. This condition is satisfied, for example, whenever the holonomy group is cyclic. If $X$ is a flat Hermitian manifold, the condition is satisfied if the holonomy group is abelian.

If we omit the orientability assumption on $X$ we find that the same condition is sufficient to insure $(n-1)$-parallelizability, i.e., the existence of a field of orthonormal $(n-1)$-frames on $X$, where $n$ is the dimension of $X$.

1. Recall that a Lie group $G$ is said to be monothetic if there exists an element $g \in G$, called a monothetic generator, whose powers are dense in $G$. We shall say that a Lie subgroup $H$ of a Lie group $G$ is submonothetic in $G$ if there exists a monothetic subgroup $H^{\prime}$ of $G$ containing $H$. Every abelian subgroup of the group of unitary transformations on a complex Hermitian vector space is submonothetic since each such is contained in a maximal torus.

Let $X$ be a smooth (i.e., $C^{\infty}$ ) Riemannian manifold, let $x \in X$, and let $H$ be the holonomy group of $X$ at $x . H$ is then a subgroup of the group of orthogonal transformations on the tangent space $X(x)$ to $X$ at $x$ and we shall say that $H$ is submonothetic if it is submonothetic in this group.

Our main theorem, to be proved in $\S 3$, is as follows. By "flat" we mean "with zero curvature."

Theorem 1. Let $X$ be a flat Riemannian manifold of dimension $n$ with submonothetic holonomy group. Then $X$ is $(n-1)$-parallelizable. If further $X$ is orientable, then $X$ is in fact parallelizable.

A description of a class of compact manifolds satisfying the hypotheses of this theorem, namely those with holonomy group cyclic of prime order, has recently been given by L. S. Charlap [3].

Presented to the Society, February 29, 1964; received by the editors September 5, 1963.

${ }^{1}$ This research was partially supported by an N.S.F. Cooperative Graduate Fellowship at Columbia University and partially by the Air Force Office of Scientific Research. 
COROLlARY. Let $X$ be a flat Hermitian manifold with abelian holonomy group. Then $X$ is parallelizable.

Proof. Since $X$ is Hermitian, it is orientable. Further, the holonomy group $H$ at $x \in X$ is then a subgroup of the group of unitary transformations on $X(x)$ and since $H$ is abelian it is submonothetic in this group. If we regard $X$ now as a flat Riemannian manifold, the unitary transformations on $X(x)$ form a subgroup of the group of orthogonal transformations and hence $H$ is submonothetic here. Applying the theorem completes the proof.

2. In order to prove Theorem 1, we need the following result about arbitrary Riemannian manifolds with submonothetic holonomy groups.

THEOREM 2. Let $X$ be Riemannian with submonothetic holonomy group. Then the tangent bundle of $X$ admits a decomposition as an orthogonal Whitney sum of invariant (under parallel translation) orientable 2-plane bundles and invariant line bundles. The line bundles in the decomposition are trivial except possibly one, which is trivial if and only if $X$ is orientable.

Proof. Let $g$ be a monothetic generator of the monothetic group containing the holonomy group $H$ of $X$ at $x \in X$. Relative to an appropriate orthonormal frame at $x, g$ is represented by a matrix of the form

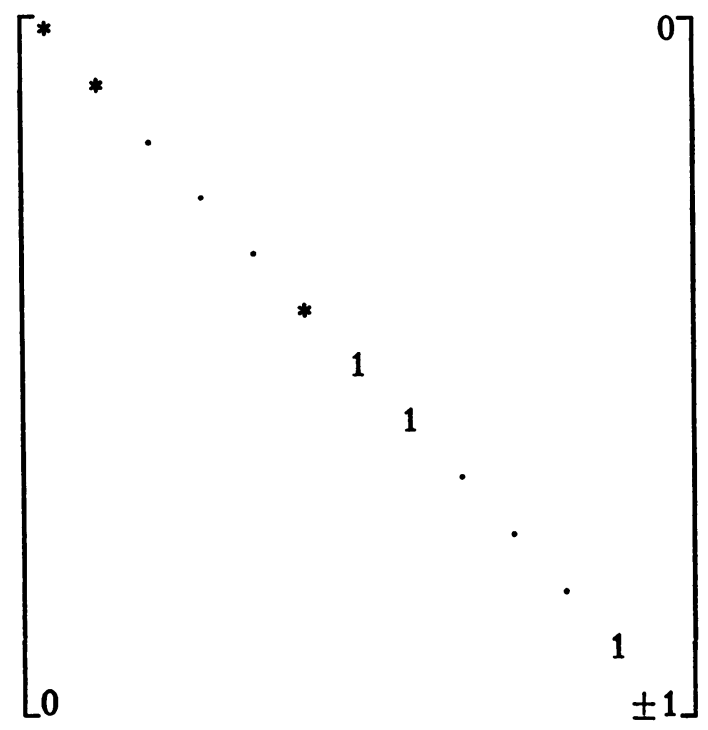

where each $*$ is a $2 \times 2$ matrix of the form 


$$
\left[\begin{array}{ll}
\cos \theta & \sin \theta \\
-\sin \theta & \cos \theta
\end{array}\right]
$$

and where the sign of the last entry is + or - depending on whether $X$ is orientable or nonorientable. (Note that in the usual canonical form there may be several -1's on the diagonal but we prefer to group them by pairs into a * with $\theta=\pi$.) Thus the tangent space to $X$ at $x$ admits an orthogonal decomposition as a sum of oriented twodimensional subspaces, oriented lines, and possibly an unoriented line, which are invariant under $g$, hence under $H$. Thus parallel translation of these subspaces is well defined on $X$ and gives rise to the required Whitney sum decomposition of the tangent bundle of $X$.

COROllary. Let $X$ be Riemannian of dimension $n$ with submonothetic holonomy group. A ssume that the second integral cohomology group $H^{2}(X ; Z)$ of $X$ is zero. Then $X$ is $(n-1)$-parallelizable. If further $X$ is orientable, then $X$ is in fact parallelizable.

Proof. It suffices to show that each of the 2-plane bundles in the decomposition of Theorem 2 is trivial. But an orientable 2-plane bundle $\xi$ is trivial if and only if its Euler class is zero. Since the Euler class is an element of $H^{2}(X ; Z)$, it is zero by assumption.

3. Proof of Theorem 1. We shall use the well-known fact that the null-holonomy group of a flat manifold is trivial, i.e., the only element of the holonomy group arising from curves homotopic to zero is the identity element. This is an immediate consequence of the holonomy theorem [1], using the fact that the null-holonomy group is the arcwise connected component of the identity in the holonomy group. Thus, for $X$ flat, parallel translation over simply connected domains is well defined, independent of curve.

Now let the orientable 2-plane bundles in the decomposition of Theorem 2 each be given a definite orientation. It suffices to show that for each 2-plane bundle $\xi$ we can define a continuous field of orthonormal 2-frames which at each $x \in X$ spans the fiber $p(x)$ of $\xi$ at $x$. (Such a field may then, of course, be approximated by a smooth one.) Note that $p: X \rightarrow G_{2}(X)$ is a section of the Grassmann bundle $G_{2}(X)$ of oriented 2-planes tangent to $X$.

We may assume (cf. [4]) that $X$ is given a smooth triangulation. For each vertex $e_{\alpha}$, let $f\left(e_{\alpha}\right)$ be any orthonormal 2-frame spanning $p\left(e_{\alpha}\right)$ and determining its orientation. We now extend $f$ to a continuous section, also denoted by $f$, in the bundle $F_{2}(X)$ of orthonormal 2 -frames on $X$ in such a way that $\rho \circ f=p$, where $\rho: F_{2}(X) \rightarrow G_{2}(X)$ is the natural projection. For this, let $\sigma$ be an $n$-simplex of $X$, with 
vertices $e_{0}, \cdots, e_{n}$ say. For $x \in \sigma$, let $\lambda_{i}(x)(0 \leqq i \leqq n)$ denote the barycentric coordinates of $x$ in $\sigma$, i.e., $x=\sum_{i=0}^{n} \lambda_{i}(x) e_{i}, \lambda_{i}(x) \geqq 0$, and $\sum_{i=0}^{n} \lambda_{i}(x)=1$. Now let $f^{\prime}(x)$ be the parallel translate to $x$ of the frame $f\left(e_{0}\right)$, along any curve in $\sigma . f^{\prime}(x)$ is well defined since $\sigma$ is simply connected and $X$ is flat. Now, since $\xi$ is invariant under parallel translation, $\rho\left(f^{\prime}\left(e_{i}\right)\right)=p\left(e_{i}\right)=\rho\left(f\left(e_{i}\right)\right)$ for $0 \leqq i \leqq n$. Thus, for $0 \leqq i \leqq n$, there exists an angle $\theta_{i}$ with $0 \leqq \theta_{i}<2 \pi$ such that

$$
f\left(e_{i}\right)=R\left(\theta_{i}\right) f^{\prime}\left(e_{i}\right),
$$

where $R\left(\theta_{i}\right)$ denotes rotation in the oriented plane $p\left(e_{i}\right)$ through the angle $\theta_{i}$. For $x \in \sigma$ we define

$$
f_{\sigma}(x)=R\left(\sum_{j=0}^{n} \lambda_{j}(x) \theta_{j}\right) f^{\prime}(x) .
$$

Clearly $f_{\sigma}$ agrees with $f$ on the verticès of $\sigma$. Further, $f_{\sigma}$ is smooth on $\sigma$ and satisfies $\rho \circ f_{\sigma}=p$.

We claim that the definition of $f_{\sigma}$ is independent of the vertex $e_{0}$ used in defining it. In fact, suppose we follow the same procedure using another vertex of $\sigma$, which we may without loss of generality assume is $e_{1}$. For $x \in \sigma$, let $f^{\prime \prime}(x)$ be the parallel translate to $x$ of $f\left(e_{1}\right)$ and let $\beta_{i}(0 \leqq i \leqq n)$ be such that $0 \leqq \beta_{i}<2 \pi$ and

$$
f\left(e_{i}\right)=R\left(\beta_{i}\right) f^{\prime \prime}\left(e_{i}\right) .
$$

Then $\beta_{i}=\theta_{i}-\theta_{1}(0 \leqq i \leqq n)$ and $f^{\prime \prime}(x)=R\left(\theta_{1}\right) f^{\prime}(x)$ for $x \in \sigma$ so that

$$
\begin{aligned}
R\left(\sum \lambda_{j}(x) \beta_{j}\right) f^{\prime \prime}(x) & =R\left(\sum \lambda_{j}(x) \theta_{j}-\theta_{1}\right) R\left(\theta_{1}\right) f^{\prime}(x) \\
& =R\left(\sum \lambda_{j}(x) \theta_{j}\right) f^{\prime}(x)
\end{aligned}
$$

for all $x \in \sigma$, completing the proof of the claim. Since the definition of $f_{\sigma}$ is symmetric in $e_{1}, \cdots, e_{n}$ it now follows that $f_{\sigma}$ is independent of the choice of labelling for the vertices of $\sigma$.

We now show that if $\sigma_{1}$ and $\sigma_{2}$ are $n$-simplexes with a common face $\tau$, then the definitions of $f_{\sigma_{1}}$ and $f_{\sigma_{2}}$ agree on $\tau$. In fact, by the above argument, we may assume that the first $n$ vertices both of $\sigma_{1}$ and of $\sigma_{2}$ are the vertices $e_{0}, \cdots, e_{n-1}$ of $\tau$. Then, for $x \in \tau$, the $(n+1)$ st barycentric coordinates of $x$ in $\sigma_{1}$ and $\sigma_{2}$ are zero and

$$
f_{\sigma_{1}}(x)=R\left(\sum_{i=1}^{n-1} \lambda_{i}(x) \theta_{i}\right) f^{\prime}(x)=f_{\sigma_{2}}(x)
$$

where the $\lambda_{i}(x)$ may be regarded as the barycentric coordinates of $x$ in $\tau$. Thus the $f_{\sigma}$ agree on common faces and define a continuous section $f: X \rightarrow F_{2}(X)$ with $\rho \circ f=p$, completing the proof. 
REMARK 1. As L. Charlap has pointed out, the proofs of Theorems 1 and 2 extend easily to prove the following. Let $X$ be a flat Riemannian manifold. Let $T_{c}(X)$ denote the complexification of the tangent bundle of $X$. Then the holonomy group $H$ of $X$ is in a natural way a subgroup of the structural (unitary) group of $T_{C}(X)$. If $H$ is submonothetic as a subgroup of this unitary group then the bundle $T_{C}(X)$ is trivial. As a consequence, all Pontrjagin classes (with arbitrary coefficients) of such an $X$ are zero. This remark applies in particular to all flat Riemannian manifolds with abelian holonomy groups.

REMARK 2. It is well known that flat Riemannian manifolds and solvmanifolds (homogeneous spaces of solvable Lie groups) have many properties in common. In this direction it is interesting to compare the above results with those recently obtained for solvmanifolds by L. Auslander and R. H. Szczarba [2].

\section{REFERENCES}

1. W. Ambrose and I. M. Singer, A theorem on holonomy, Trans. Amer. Math. Soc. 75 (1953), 428-443.

2. L. Auslander and R. H. Szczarba, Characteristic classes of compact solvmanifolds, Ann. of Math. (2) 76 (1962), 1-8.

3. L. S. Charlap, Compact flat Riemannian manifolds. I (to appear).

4. J. R. Munkres, Elementary differential topology, Annals of Mathematics Studies No. 54, Princeton Univ. Press, Princeton, N. J., 1963.

Massachusetts Institute of Technology 